\title{
Wavelet-based index of magnetic storm activity
}

\author{
A. Jach, ${ }^{1}$ P. Kokoszka, ${ }^{1}$ J. Sojka, ${ }^{2}$ and L. $Z^{2}{ }^{2}$ \\ Received 26 January 2006; revised 21 April 2006; accepted 27 June 2006; published 22 September 2006.
}

[1] A wavelet-based method of computing an index of storm activity is put forward.

The new index can be computed automatically using statistical procedures and does not require selecting quiet days and removing the secular component by polynomial fitting. This 1-min index is designed to facilitate the study of the fine structure of geomagnetic storm events and requires only the most recent magnetogram records, e.g., the 2 months including the storm event of interest. It can thus be computed over a moving window as soon as new magnetogram records become available. Averaged over 1-hour periods, it is practically indistinguishable from the standard Dst index.

Citation: Jach, A., P. Kokoszka, J. Sojka, and L. Zhu (2006), Wavelet-based index of magnetic storm activity, J. Geophys. Res., 111, A09215, doi:10.1029/2006JA011635.

\section{Introduction}

[2] The currents flowing in the magnetosphereionosphere (M-I) form a complicated multiscale geosystem that contains the temporal scales from seconds to days. Ground-based magnetometers have long been an important tool to observe the M-I current system and a number of indices based on magnetometer data have been introduced to characterize the variations of specific current components. Because of the nature of this current system, the magnetometer data are multiscale, impulsive, and asynchronous with nonstationary frequency spectra. On the basis of the assumption that magnetometers in certain latitude bands are most sensitive to specific currents, a traditional way to separate the magnetic effects of different currents is to use magnetometer records from a specific region and combine them into an index characterizing the variation of a specific current. References to review papers on geomagnetic indices are given by Kivelson and Russell [1997, p. 409], and they present a concise account of the main indices [Kivelson and Russell, 1997, Appendix 13B, p. 451].

[3] In this paper we propose an automatic wavelet-based statistical procedure designed to develop an index of storm activity associated with the intensification of the ring current. The Dst index [see Sugiura, 1964] has long been used to characterize this variation and is produced from the magnetometer data recorded in the equatorial region. The Dst index was originally designed to describe the variation of the symmetric ring current. However, the procedure of producing the Dst index cannot eliminate the magnetic effects from many local-time-dependent currents in the ionosphere and magnetosphere, including the partial ring current, auroral currents, magnetotail current, etc. Therefore, what the Dst index describes is actually the overall

\footnotetext{
${ }^{1}$ Department of Mathematics and Statistics, Utah State University, Logan, Utah, USA.

${ }^{2}$ Department of Physics and Center for Atmospheric and Space Science, Utah State University, Logan, Utah, USA.
}

Copyright 2006 by the American Geophysical Union. 0148-0227/06/2006JA011635\$09.00 magnetic effect of storm activity at the low-latitude and midlatitude regions (see). A main difficulty in producing this index lies in subtracting the quiet day variation from a magnetogram recorded at a given location. After this quiet day component and the long-term component have been subtracted, the remainder is believed to describe the storm related magnetic activity. Constructing the quiet day component involves somewhat subjectively choosing several days in a month, during which the storm activity is believed to be absent, and averaging them. We show that using a wavelet technique it is possible to produce a 1-min index which, after averaging over one hour periods, is very close to the standard Dst. The unique strength of the technique we propose is that it is fully automatic and, in particular, does not involve selecting quiet days. In this technique, the daily and long-term variation, as well as inconsequential noise, are removed in the wavelet domain by means of statistical filtering. The procedure requires only the most recent stretch of data, and can be used to quickly produce a signature of a storm event.

[4] We now introduce a wavelet-based representation for magnetometer data and formulate a time series model needed to explain the central ideas of the proposed approach. More details are presented in section 2. For the purpose of this study, the specific magnetogram record to be used is the station's H component, i.e., the magnitude of the horizontal magnetic field. This is the same component as used in the standard Dst calculation [see Sugiura, 1964].

[5] Suppose $\mathbf{X}_{s}=\left\{X_{s, t}: t=0,1, \ldots, N-1\right\}$ is a magnetogram recorded at station $s$, the sample size $N$ is the length of the record in minutes. The magnetogram $\mathbf{X}_{s}$ can be decomposed as

$$
\mathbf{X}_{s}=\sum_{j=1}^{J} \mathbf{D}_{s, j}+\mathbf{S}_{s, J}
$$

where

$$
\mathbf{D}_{s, j}=\left\{D_{s, j, 0}, \ldots, D_{s, j, N-1}\right\}, \quad \mathbf{S}_{s, J}=\left\{S_{s, J, 0}, \ldots, S_{s, J, N-1}\right\} .
$$


Decomposition (1) is known as the multiresolution analysis (MRA). The details $D_{s, j, t}$ correspond to the component of the record at time of approximately $2^{j} t$ minutes and to frequencies in the range of $2^{-j-1}$ to $2^{-j}$ cycles per minute. This range corresponds to physical scales between $2^{j} / 60$ and $2^{j+1} / 60$ hours. The smooths $S_{s, J, t}$ correspond to time of approximately $2^{J} t$ minutes and to frequencies lower than $2^{-J-1}$ cycles per minute, or, alternatively, to broadly understood averages over intervals of $2^{J+1} / 60$ hours.

[6] As will be seen in the following, the different components of the magnetogram are most prominent at specific levels $j$ of decomposition (1). By applying suitable statistical filters to each level $j$, we are able to isolate and remove the components which do not reflect the storm activity. It should be noted that the following procedure still cannot separate and distinguish the magnetic effects associated with several local time-dependent currents (for example, partial ring current and auroral currents).Therefore resulting wavelet-based index basically describes the variations of the same physical processes as the Dst index does, that is the storm time activity. However, the important new features of the wavelet index, which are not encountered in the conventional $D s t$, are automation (with no human interference) and flexibility on the data stretch.

[7] To justify our methodology, we assume the following approximate model for the time series $\left\{X_{s, t}\right\}$ :

$$
X_{s, t}=B_{s}+A_{s, t}+P_{s, t}+L_{s, t}+N_{s, t},
$$

where $B_{s}$ is internal magnetic field of the Earth measured at a low-latitude station $s$ (assumed to be time-independent), $A_{s, t}$ is disturbance component attributable to storm activity, $P_{s, t}$ is periodic component, $L_{s, t}$ is slowly varying trend (assumed to be a low degree polynomial), and $N_{s, t}$ is noise component.

[8] The components $A_{s, t}$ and $N_{s, t}$ should be thought of as random; $A_{s, t}$ is a "large" random component which becomes pronounced during a storm; $N_{s, t}$ is a "small" random component reflecting all kinds of random disturbances from the measurement error to irregular random disturbances of the M-I system. Small random variations in deterministic components $B_{s}, P_{s, t}$ and $L_{s, t}$ are thought of as being moved to $N_{s, t}$, so, for example, $P_{s, t}$ is considered a deterministic periodic component to be statistically estimated. By contrast, the component $A_{s, t}$, which we want to isolate, is random because we do not assume anything about the timing or signature of a storm event.

[9] Admittedly, model (2) is a simplification needed to develop a usable statistical methodology. For example, $L_{s, t}$ evolves in a complex manner with the solar cycle and treating it as a polynomial is only an approximation. The periodicity of $P_{s, t}$ is only a mathematical assumption; $P_{s, t}$ should not be identified with the simple diurnal variability, a loose association is however helpful to understand the procedure. Without these assumptions, the components on the right-hand side would not be identifiable, as only the $X_{s, t}$ are observable and five components cannot be uniquely identified from their sum. The mathematical assumptions thus play a role of additional equations. A further caveat is that the present analysis does not make any corrections for the ground induction effects. The temporary variations of various components of induction currents are similar to their source currents. Therefore their effect on an index is believed not to be significant. Our omission of this correction is in keeping with the existing procedures for calculating the Dst index.

[10] The paper is organized as follows. In section 2 we present some background the wavelet analysis. Sections 3 and 4 focus on the removal of the noise and periodic components, respectively. In section 5, we formulate the procedure for computing our wavelet based index, and in section 6 we compare it to the standard Dst index. We conclude with final remarks in section 7 .

\section{MODWT of the Magnetometer Data}

[11] In this section we describe the maximum overlap discrete wavelet transform (MODWT) and explain how it can be applied to a single magnetogram component (scalar data) from a single station. We will also point out the advantages of the MODWT over the discrete wavelet transform (DWT). Finally, we will discuss the choice of the parameters of the wavelet analysis. Our choices are motivated by the task of isolating storm signatures in the presence of a strong periodic component.

[12] Throughout this section, we follow closely the exposition and notation of Percival and Walden [2000]. We focus on the aspects of the MODWT which are most relevant to our task, and cannot present all details. An interested reader is referred to Percival and Walden [2000, chapter 5].

[13] The MODWT is a nonorthogonal modification of the DWT which addresses some shortcomings of the latter, such as sample size restriction and sensitivity to the starting point of the series. Like the DWT, the MODWT produces a set of wavelet and scaling coefficients obtained by linear filtering of the signal (this is done by an efficient pyramid algorithm, although with a somewhat higher computational burden than that for the DWT). Unlike the DWT, there are $N$ coefficients at each scale, where $N$ is the number of observations. The MODWT details and smooths are associated with zero phase filters and, unlike details and smooths of the DWT, do not require any shifting to align time events.

[14] Suppose $\mathbf{X}_{s}=\left\{X_{s, t}: t=0,1, \ldots, N-1\right\}$ is a scalar component of a magnetogram recorded at station $s$. For any integer $1 \leq j \leq \log _{2}(N)$, the MODWT wavelet and scaling coefficients at level $j$ are defined as

$$
W_{s, j, t}=\sum_{l=0}^{L_{j}-1} \tilde{h}_{j, l} X_{s, t-l} \bmod N
$$

and

$$
V_{s, j, t}=\sum_{l=0}^{L_{j}-1} \tilde{g}_{j, l} X_{s, t-l \bmod N},
$$

where $t=0,1, \ldots, N-1$, and $\left\{\tilde{h}_{j, l}\right\}$ and $\left\{\tilde{g}_{j, l}\right\}$ are the $j$ th level MODWT wavelet and scaling filters, both of length $L_{j}=$ $\left(2^{j}-1\right)(L-1)+1 ; L$ denotes the length of the underlying wavelet filter, for example, the Daubechies $\mathrm{D}(L)$ or the least asymmetric LA $(L)$ filter [see Percival and Walden, 2000, section 4.8]. The " $\bmod N$ " indicates circular convolution [see Percival and Walden, 2000, section 2.5]. In practice, 
Level 8

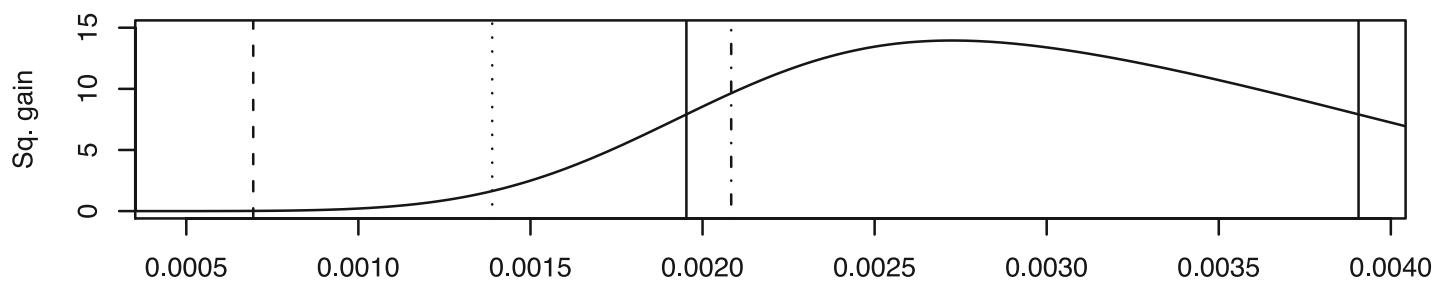

\section{Level 9}

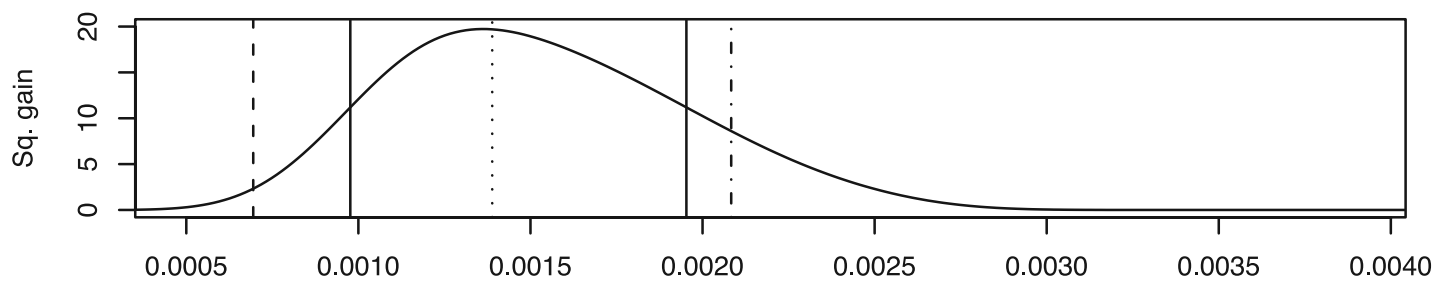

Level 10

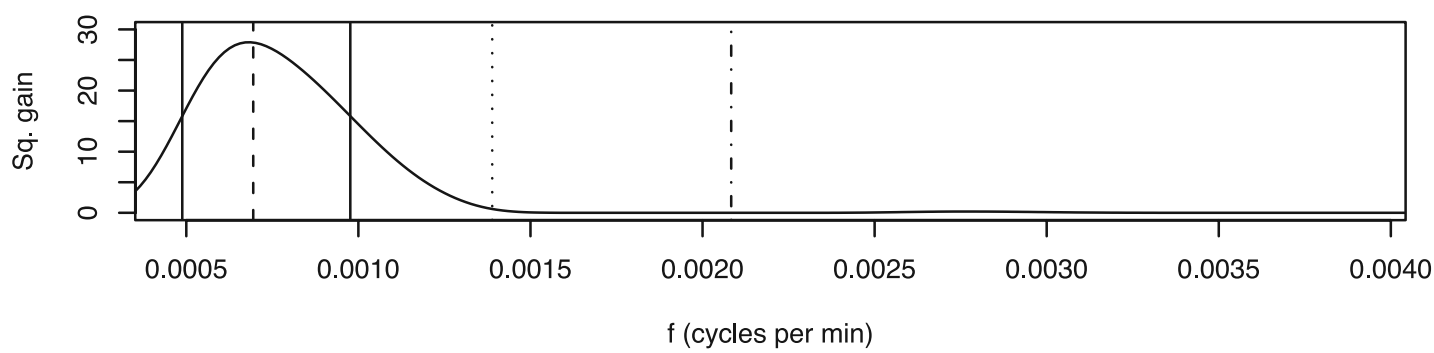

Figure 1. Squared gain functions $\widetilde{\mathcal{H}}_{j, L}(f)$ for $\operatorname{LA}(8)$ filter, levels $j=8,9,10$. Nominal passbands are indicated by solid vertical lines. Periodic frequencies 1/1440, 2/1440, and 3/1440 are marked by dashed, dotted, and dash-dotted lines, respectively.

because of the application of the pyramid algorithm, these filters need not be computed.

[15] The transfer function $\widetilde{H}_{j, L}(f)$ for the level $j$ filter can be regarded as an approximation to a transfer function of a perfect band-pass filter with passband $\left[1 / 2^{j+1}, 1 / 2^{j}\right]$. The squared gain functions, defined as $\widetilde{\mathcal{H}}_{j, L}(f)=\left|\widetilde{H}_{j, L}(f)\right|^{2}$, are shown in Figure 1, which will be referred to later. Level $j$ wavelet coefficients are thus associated with the portion of discrete Fourier transform (DFT) of $\mathbf{X}_{s}$ with frequencies in the interval $\left[1 / 2^{j+1}, 1 / 2^{j}\right]$.

[16] The MODWT yields decomposition (1) with level $j$ detail and smooth sequences $\mathbf{D}_{s, j}$ and $\mathbf{S}_{s, j}$ defined by

$$
D_{s, j, t}=\sum_{l=0}^{L_{j}-1} \tilde{h}_{j, l} W_{s, j, t+l \bmod N}, \quad t=0,1, \ldots, N-1
$$

and

$$
S_{s, j, t}=\sum_{l=0}^{L_{j}-1} \tilde{g}_{j, l} V_{s, j, t+l \bmod N}, \quad t=0,1, \ldots, N-1
$$

[17] The circular $(\bmod N)$ filtering in (3)-(4) and $(5)-(6)$ generates the so-called boundary MODWT coefficients which do not have a physical interpretation, but are due to the fact that the observations are not part of an infinite sequence with period $N$. For long records, there are relatively few boundary coefficients, especially for small $j$. Several methods of reducing the impact of circular filtering have been devised. In this paper we use the reflection rule [see Percival and Walden, 2000, pp. 140-141].

[18] The application of the MODWT to the magnetometer data requires additional specifications, such as the choice of a wavelet filter and the maximum level $J$. With the goals of the analysis in mind, we discuss these issues below.

\subsection{Wavelet Filter}

[19] In our study, we use the LA(8) filter which has been extensively applied in several recent quantitative analyzes of geophysical data [see, e.g., Whitcher et al., 2002; Overland et al., 2004; Cornish et al., 2004]. Using Daubechies filter $\mathrm{D}(8)$ produces the same results. LA filters are 
parameterized by even widths $L$. For a specific analysis, a width must be selected which would provide sufficient concentration in the octave passband and reduce the overlapping of frequencies from outside of it. This can be achieved by selecting a relatively long filter, but choosing $L$ too large will result in many boundary coefficients, especially at higher levels $j$. Choosing $L=8$ has been found to offer a reasonable balance between these two competing requirements. This choice also guarantees that the MODWT coefficients are uninfluenced by a polynomial of order $K \leq$ $L / 2-1=3$. Thus a slowly varying trend (secular variation) in the magnetic field, which is typically modeled by a loworder polynomial [see Kivelson and Russell, 1997, p. 457] is practically not reflected in the details of the MODWT of magnetometer data. On the other hand, for 1-year-long magnetometer data, this trend can be captured by a smooth $\mathbf{S}_{S, J}$ on the highest available level, and thus an appropriate adjustment of $\mathbf{S}_{S, J}$ will automatically remove it.

\subsection{Number of Levels $J$}

[20] The maximum level $J$ in (1) is typically chosen to be smaller than its largest admissible value $\left\lfloor\log _{2}(N)\right\rfloor$. However, for 1-year-long data, we need to account for the secular variation. Decomposing the signal up to level $J=\left\lfloor\log _{2}(N)\right\rfloor=$ 19 and replacing $\mathbf{S}_{19}$ by its average (this average is equal to the average of the magnetogram) allow to eliminate the annual trend. For shorter records, for example, 2-month long, the smooth corresponding to the highest available level is affected by the storm events and cannot be regarded as a good approximation to the annual trend. We assume that the longterm component is not visible in the records extending over the period of 2 months. This assumption is validated by comparing our index computed from 2 months of data to the standard Dst index. For such records we propose to set $J=10$, because $J$ should be large enough to allow the removal of the noise and periodic components. Since the latter is visible at higher scales, $J$ will be dictated by the frequencies contributing to the periodic $(\mathrm{Sq})$ variation. As we show in section 4, the lowest frequency corresponding to the periodic portion of the magnetogram is the daily frequency, $1 / 1440$, that falls in $\left[2^{-j-1}, 2^{-j}\right], j=10$, but there are also some higher frequencies. Taking $J=10$ ensures that these frequencies can be effectively removed.

\section{Noise Removal via Wavelet Thresholding}

[21] Wavelet thresholding is a nonparametric method of estimating a signal in the presence of additive noise (see Percival and Walden [2000, chapter 10] for a review) and has been developed both theoretically and practically over the last decade. The underlying assumption of this methodology is that the noise is "small" compared to the signal. We use thresholding to remove the component $N_{s, t}$ in decomposition (2). To justify this approach, note that by (2) and (3)

$$
W_{s, j, t}=W_{s, j, t}^{(A)}+W_{s, j, t}^{(P)}+W_{s, j, t}^{(N)}
$$

where the $W_{S, j, t}^{(A)}$ are the MODWT coefficients of the storm component $A_{s, t}, W_{s, j, t}^{(P)}$ and $W_{s, j, t}^{(N)}$ being defined analogously. The wavelet coefficients of the components $B_{s}$ and $L_{s, t}$ vanish because these components are assumed to be, respectively, a constant and a polynomial of degree not exceeding three. At low levels $j \leq J_{0}$, where the value of $J_{0}$ is specified at the end of this section, the periodic coefficients $W_{s, j, t}^{(P)}$ are negligible; see section 4 . Thus we may, in fact, assume that

$$
W_{s, j, t}=W_{s, j, t}^{(A)}+W_{s, j, t}^{(N)}
$$

Moreover, for $j \leq J_{0}$, the $W_{s, j, t}$ are mostly "small noise" with large coefficients during storm events. The goal of thresholding is to remove the $W_{s, j, t}^{(N)}$ which constitute the vast majority of the coefficients at low scales, and to estimate $W_{s, j, t}^{(A)}$. This can be done only in some statistical sense because the $W_{s, j, t}^{(N)}$ are not observable. We assume that the absolute values of the $W_{s, j, t}^{(N)}$ approximately do not exceed a level $\delta_{j}$. The observed coefficients $W_{s, j, t}$ are thus replaced by zero if their absolute value is smaller than $\delta_{j}$ and their length is reduced by $\delta_{j}$ if their absolute value is greater than $\delta_{j}$. The resulting coefficients are denoted by $\bar{W}_{s, j, t}$ and are thus given by

$$
\begin{gathered}
\bar{W}_{s, j, t}=\operatorname{sign}\left\{W_{s, j, t}\right\}\left(\left|W_{s, j, t}\right|-\delta_{j}\right)_{+}, \\
t=0,1, \ldots, N-1 \quad\left(j \leq J_{0}\right) .
\end{gathered}
$$

The $\bar{W}_{s, j, t}$ are statistical estimates of the $W_{s, j, t}^{(A)}$ in the absence of the $W_{s_{j}, t}^{(P)}$.

[22] Formula (9) defines the so-called soft level-dependent thresholding. In hard thresholding, coefficients $W_{s, j, t}$ whose absolute value exceeds $\delta_{j}$ are not modified. Soft thresholding seems more appropriate in our context because formula (8) suggests that $W_{s, j, t}^{(N)}$ should be removed from all coefficients. Moreover, our exploratory analysis has shown that hard thresholding introduces occasional long spikes to the proposed index of storm activity which have no reasonable physical interpretation. In level-independent thresholding $\delta_{j}=\delta$ is the same for all levels and is typically determined by the statistical behavior of the coefficients at the finest level $j=1$. This approach is suitable for the task of removing noise from a signal, but is not appropriate in our context because for magnetometer data the magnitude of the $W_{s, j, t}^{(N)}$ visibly changes with level $j$.

[23] A natural and convenient way to select $\delta_{j}$ is to define it as the $p$ th quantile of the distribution of the absolute values of the $W_{s, j, t}$. Thus we define $\delta_{j}=\delta_{j, s}(p)$ by the formula

$$
P\left(\left|W_{s, j, t}\right| \leq \delta_{j, s}(p)\right)=p
$$

The probability $P$ on the left-hand side of (10) is the empirical probability, i.e., the proportion of the $\left|W_{s, j, t}\right|, t=0,1, \ldots$, $N-1$ which exceed $\delta_{j, s}(p)$. Our exploratory analysis has shown that $p$ should be taken relatively large (close to 1$)$. For example, if $p=0.98$, all coefficients, except the largest (in absolute terms) $2 \%$ of are set to zero. Note that this statistical approach guarantees that the thresholding can be automatically applied to every station no matter what the typical magnitude for the magnetic field is.

[24] Recall that $J_{0}$ is the largest scale on which the effect of the periodic component is negligible. The periodic 
component is associated with the Sq variation which has a pronounced daily frequency (1/1440 cycles per minute), halfdaily frequency $(2 / 1440)$ and whose spectrum also contains higher frequencies, even though these are much less pronounced. Note that the half-daily frequency corresponds to the scale of $1440 / 2=770 \mathrm{~min}$ which is contained in the scale range $[512,1024]$ corresponding to $j=9$. Because of the presence of frequencies higher than 2/1440 in the spectrum of the $\mathrm{Sq}$ variation, the periodic component is also visible at level $j=8$. Visual inspection reveals that at levels $j \leq 7$, the periodic component is not visible and the MODWT coefficients at these levels form an approximately stationary process. We therefore choose $J_{0}=7$ based both on theoretical grounds discussed above and on an exploratory analysis of the wavelet decompositions of the magnetometer data.

\section{Removal of the Periodic Component}

[25] This section proposes a statistical method of removing the periodic component $P_{s, t}$ in decomposition (2). We loosely identify the periodic component with the daily variation caused by the rotation of the Earth. As the position of the station relative to the M-I current system changes, various currents, including the wind currents on the dayside of the ionosphere, the magnetopause and tail currents, leave approximately periodic signatures in the magnetometer data.

[26] The method will be applied to selected details $\mathbf{D}_{s, j}$ because, unlike the MODWT coefficients $W_{s, j, t}$, times $t$ in the details $D_{s, j, t}$, are aligned with times $t$ in the observations $X_{s, t}(t=0,1, \ldots, N-1)$ [see Percival and Walden, 2000, chapter 5]. Such a time alignment is not necessary in the removal of the noise which does not have a regular pattern over time. Instead, the wavelet domain equation (8) was exploited.

[27] Exploratory analysis of the details from four Dst stations, showed that the periodicity is clearly visible in the $\mathbf{D}_{s, j}$ for $j=8,9,10$ and not visible by eye at other scales. This finding agrees with the known properties of the daily variation and the spectral properties of the LA(8) wavelet filter. The daily variation [see Parkinson, 1983, section 4.4] has spectrum dominated by peaks at frequencies of 1,2 and possibly 3 cycles per day. These frequencies correspond, respectively, to wavelet levels $j=10,9,8$, as already discussed in section 3 . Figure 1 shows that the squared gain functions $\widetilde{\mathcal{H}}_{j, L}(f), j=8,9,10$, of the $\mathrm{LA}(8)$ filter together completely cover the interval [0.0005, 0.0040] which contains practically all frequencies present in the spectrum of the daily component, including the daily frequency of $1 / 1440 \approx 0.0007$ and the half-daily frequency of about 0.0014 (all frequencies are in cycles per minute).

[28] As an example, Figure 2 shows the details $\mathbf{D}_{j}$ for levels 8 through 10 for station Kakioka for March-April 2001. As we can see, the largest contribution to the periodic component comes from the "slow oscillating" details capturing the daily frequency (bottom panel) and the smallest from the "fast oscillating" $\mathbf{D}_{8}$.

[29] Recall that our goal is to extract a periodic daily component from the data. We propose to use a robust median-based filter which is analogous to the usual method of removing the periodic component [see, e.g., Brockwell and Davis, 2002, section 1.5] but uses the median instead of the average. Unlike the average, the median is not sensitive to unusually large or small observations. Moreover, the wavelet coefficients of magnetometer data have heavy tails [see Kokoszka et al., 2006]. Discussing this property here would distract us from the focus of this paper, so we merely note that for heavy-tailed observations, median-based procedures generally work better than procedures based on averaging. In our context, using the median rather than the average produces an index which is closer to the Dst index.

[30] To describe our method, suppose $Y_{0}, Y_{1}, \ldots, Y_{N-1}$ is a time series without a trend. Here, it should be thought of as the time series of details $D_{s, j, t}$ at a given level $j$ from which we wish to remove the periodic component. Recall from section 2 that the MODWT coefficients and the details do not contain a trend because the slowly varying trend in the data is modeled as a polynomial of order not exceeding 3 , and we use wavelet filter of length 8 .

[31] Given a series $Y_{0}, Y_{1}, \ldots, Y_{N-1}$ from which a periodic component with period $d$ is to be removed, we follow these steps:

[32] 1. Construct $R=\lfloor N / d\rfloor$ sequences

$$
Y_{t}, Y_{t+1 d}, Y_{t+2 d}, \ldots, Y_{t+(R-1) d}, \quad t=0,1, \ldots, d-1 .
$$

( $R$ is the number of consecutive, nonoverlapping sequences of length $d$ that "fit into" the sequence of length $N$.)

[33] 2. For each such sequence compute the median $m_{t}, t=$ $0,1, \ldots, d-1$.

[34] 3. For $t>d-1$, write $t=K d+u$, for some integers $K \geq$ 1 and $0 \leq u \leq d-1$, and set $m_{t}=m_{u}$. (In this step the sequence $m_{t}, t=0,1, \ldots, d-1$, is extended periodically to a sequence of length $N$.)

[35] 4. Compute the mean $\mu=N^{-1} \sum_{t=0}^{N-1} m_{t}$ and return sequence $\left\{Y_{t}-\left(m_{t}-\mu\right)\right\}$. (This step ensures that the estimated periodic component $m_{t}-\mu, t=0,1, \ldots, N-1$, has mean zero.)

[36] In the estimation of the periodic component we include the boundary coefficients, but since the above procedure is robust to atypical observations, it is not affected by these coefficients.

[37] In section 5, to remove the daily variation, we apply the above procedure to the details $\mathbf{D}_{s, 8}, \mathbf{D}_{s, 9}, \mathbf{D}_{s, 10}$ with periods $d=480,770,1440$, respectively.

\section{Index Algorithm}

[38] We now describe the complete algorithm for obtaining an index of storm activity which incorporates the statistical procedures described in sections $1-4$. In light of the motivation and objectives outlined in section 1 , our primary focus is on an algorithm which uses 2 months worth of data, but we also show how to construct an index over a period of 1 year.

[39] Recall that for $s=1,2, \ldots, S, \mathbf{X}_{s}=\left\{X_{s, t}: t=0,1, \ldots\right.$, $N-1\}$ is a magnetogram recorded at station $s$.

[40] In step 1, if $N$ corresponds to 2 months set $J=10$, if $N$ corresponds to 1 year, set $J=19$. In both cases, set $J_{0}=7$. Compute the MODWT coefficients $W_{s, j, t}, j=1,2, \ldots, J$, and $V_{s, J, t}$.

[41] In step 2, threshold the wavelet coefficients $W_{s, j, t}, j=$ $1,2, \ldots J_{0}=7$, to obtain $\bar{W}_{s, j, t}$ according to section 3 , and 


\section{D8}

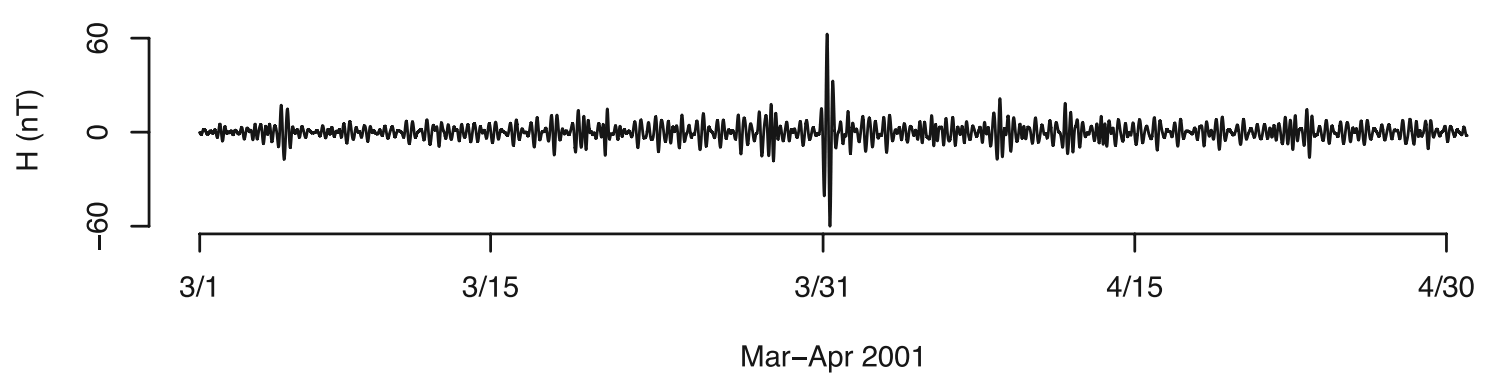

D9

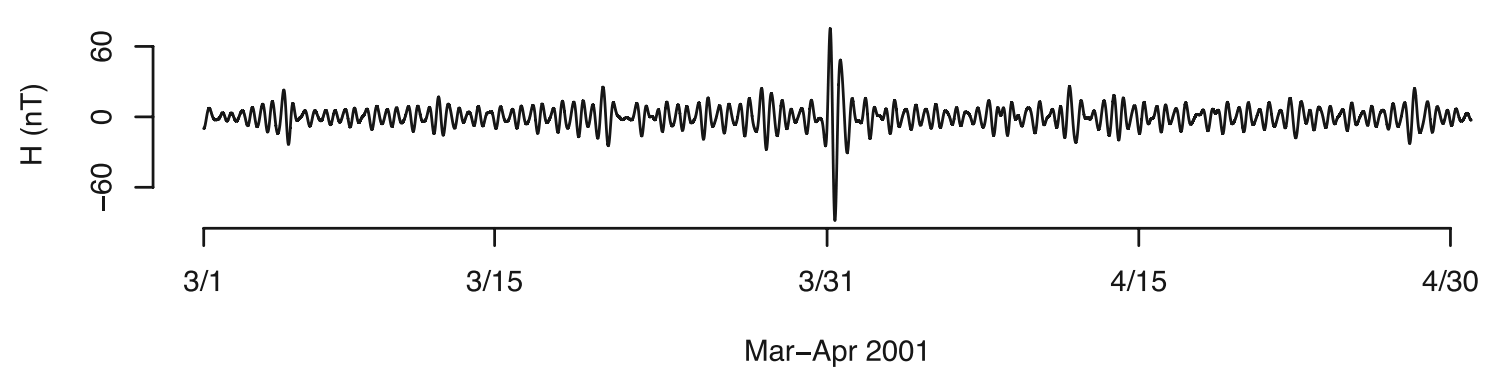

D10

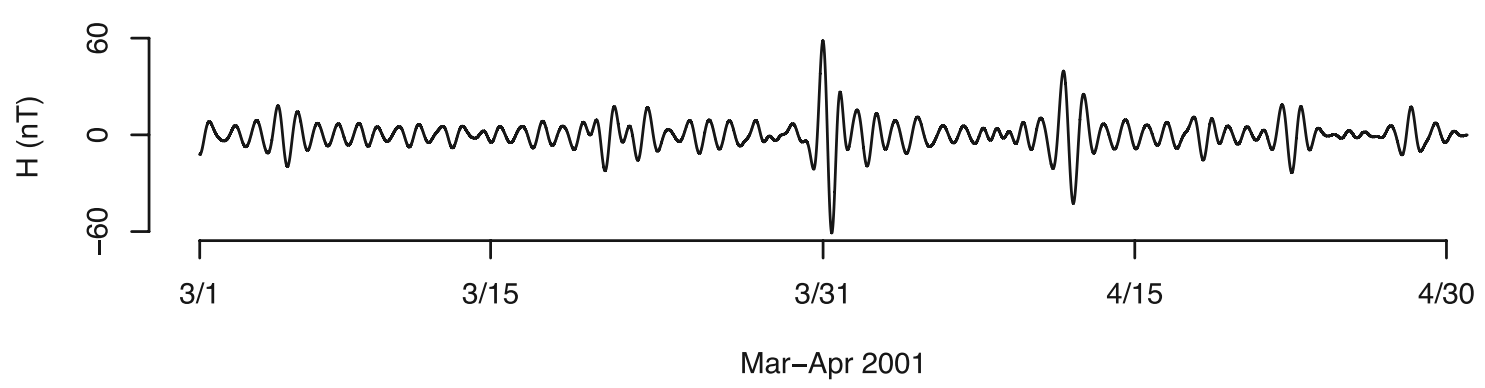

Figure 2. Details from levels $j=8,9,10$ for station Kakioka, March-April 2001.

using equation (5) compute the details $\bar{D}_{s, j, t}$ by replacing $W_{s, j, t}$ with $\bar{W}_{s, j, t}$.

[42] In step 3, compute the details $\mathbf{D}_{s, j}, j=8, \ldots, J$ and the smooth $\mathbf{S}_{s, J}$. To the details $\mathbf{D}_{s, 8}, \mathbf{D}_{s, 9}, \mathbf{D}_{s, 10}$, apply medianbased filter of section 4 with periods $d=480,770,1440$, respectively, and denote the resulting details without the periodic components by $\underline{\mathbf{D}}_{s, j}, j=8,9,10$.

[43] In step 4a, if $N$ corresponds to 2 months, compute the filtered magnetogram from which the periodic and noise components have been removed:

$$
\begin{aligned}
\widehat{A}_{s, t}+\widehat{B}_{s} & =\sum_{j=1}^{7} \bar{D}_{s, j, t}+\sum_{j=8}^{10} \underline{D}_{s, j, t}+S_{s, 10, t} \\
& =X_{s, t}-\widehat{P}_{s, t}-\widehat{L}_{s, t}-\widehat{N}_{s, t} .
\end{aligned}
$$

[44] In step 4 b, if $N$ corresponds to 1 year $(J=19)$, replace the smooth $\mathbf{S}_{s, J}$, which reflects the long-term (secular) component, by its average $N^{-1} \sum_{t=0}^{N-1} S_{S, J, t}=$
$N^{-1} \sum_{t=0}^{N-1} X_{s, t}$ and compute the filtered magnetogram from which the trend, periodic and noise components have been removed:

$$
\begin{aligned}
\widehat{A}_{s, t}+\widehat{B}_{s} & =\sum_{j=1}^{7} \bar{D}_{s, j, t}+\sum_{j=8}^{10} \underline{D}_{s, j, t}+\sum_{j=11}^{19} D_{s, j, t}+\frac{1}{N} \sum_{t=0}^{N-1} X_{s, t} \\
& =X_{s, t}-\widehat{P}_{s, t}-\widehat{L}_{s, t}-\widehat{N}_{s, t} .
\end{aligned}
$$

[45] In step 5, compute the average of the filtered magnetogram

$$
\widehat{M}_{s}=\frac{1}{N} \sum_{t=0}^{N-1}\left(\widehat{A}_{s, t}+\widehat{B}_{s}\right)=\left\{\frac{1}{N} \sum_{t=0}^{N-1} \widehat{A}_{s, t}\right\}+\widehat{B}_{s}
$$

and center the filtered data to have mean zero:

$$
\widehat{A}_{s, t}^{c}=\left(\widehat{A}_{s, t}+\widehat{B}_{s}\right)-\widehat{M}_{s}=\widehat{A}_{s, t}-\frac{1}{N} \sum_{t=0}^{N-1} \widehat{A}_{s, t} .
$$

(This step eliminates, in particular, the constant field $B_{s}$.) 
STORM ACTIVITY INDICES FOR 2001
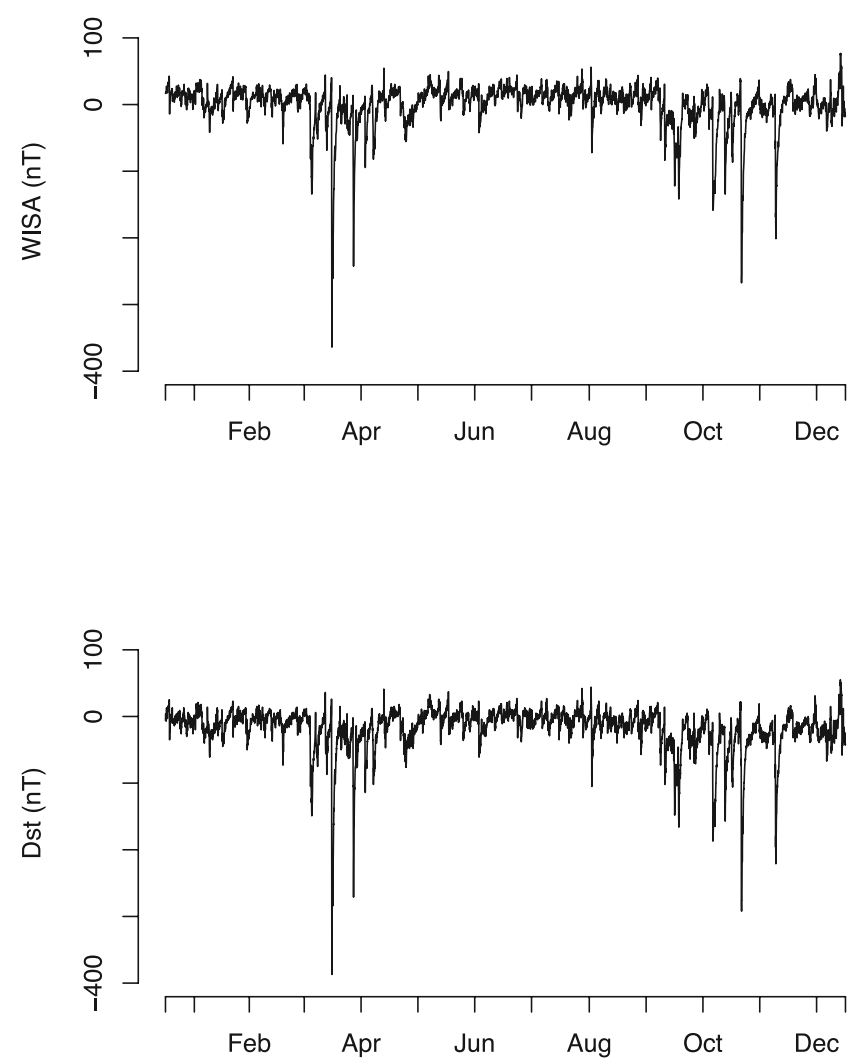

Figure 3. Hourly values of WISA and Dst, JanuaryDecember 2001.

[46] In step 6, adjust for the magnetic latitude

$$
\widehat{A}_{s, t}^{c a}=\widehat{A}_{s, t}^{c} / \cos \left(\theta_{s}\right)
$$

$\left(\theta_{s}\right.$ is the magnetic latitude of station $\left.s.\right)$;

[47] In step 7, average over all stations to obtain a global index:

$$
I_{t}=\frac{1}{S} \sum_{s=1}^{S} \widehat{A}_{s, t}^{c a}, \quad t=0,1, \ldots, N-1
$$

[48] To facilitate the discussion below, we refer to the index $I_{t}$ as the wavelet index of storm activity (WISA) in the following comments:

[49] 1. The last two steps of the algorithm are the same as for the standard Dst index [see Kivelson and Russell, 1997, p. 457]. (In the construction of the Dst index, $\widehat{A}_{s, t}^{c}$ are the data with $B_{s}, L_{s, t}$ and $P_{s, t}$ "manually" removed.)

[50] 2. In order to obtain the WISA over a given period of time, only the data from that period are used. For example, to construct an index over a period of 2 months, no observations outside that period are needed. This feature offers the flexibility of constructing the index only from new data or of using a moving window of suitably chosen length.

[51] 3. The WISA reflects only the dynamic range of storm activity over a given period of time and does not provide any reference level relative to the past. If the WISA is constructed for a period of 1 year, its mean over that year is zero, but the means over any subperiods of that year need not be zero. If the index is constructed over 2 months, its mean over these 2 months is zero.

[52] 4. The WISA can be constructed practically over any period of time, but to obtain values similar to the standard $D s t$, at least 2 months worth of data are needed. The periods of 2 months and 1 year were chosen for illustration only. Note that if $I_{t}^{1 y}$ is an index constructed from 1 year worth of data and $I_{t}^{2 m}$ is an index constructed only from, e.g., March-April data, then over the months of March and April the indices $I_{t}^{1 y}$ and $I_{t}^{2 m}$ will differ slightly.

[53] 5. Once the length of the period of time over which the WISA is to be computed is set, the procedure is fully automatic and requires merely 1 min measurements of the horizontal intensity as input.

[54] 6. Even quality 1-min data from INTERMAGNET CDs which we used contain some missing values (magnetometer data from Honolulu, $2 \%$ in 2001 and $0.1 \%$ in March-April 2001; San Juan, 2\% in 2001 and $0.3 \%$ in March-April 2001). We used linear interpolation to estimate these missing values. Linear interpolation gives slightly different values of the WISA, the difference is however not perceptible by eye on a graph showing both indices over a period of 2 months. The data we worked with had only occasional stretches of a few missing values. The presence of long stretches of missing values may be a serious problem. This issue and the use of data from non-Dst stations will be explored in future work.

[55] 7. The WISA is a $1-\mathrm{min}$ index, so in order to compare it to the standard Dst, some averaging is needed. This issue is taken up in greater detail in section 6 .

[56] 8. The algorithm described in this section can be modified in several ways while preserving the general idea. For example, different thresholding levels could be used and the removal of the periodic component could be extended to level $j=7$. Values of $p$ in the range from 0.90 to 0.99 give only a negligibly different index. At levels $j=8,9$, a component with period of 1 day could be removed rather than components with periods of, respectively, 8 and 12 hours. All these modifications would produce only a slightly different index. The algorithm we settled on seems most logical.

[57] 9. Naive removal of the details containing the noise and periodic component rather than statistical filtering produces highly oversmoothed storm events because the information about the storms is contained in all levels and must be extracted with care.

[58] 10. For 1 year data, the long-term trend at a given station is estimated by the smooth $\mathbf{S}_{s, J}$, see step $4 \mathrm{~b}$ ) of the algorithm. Since our goal is to produce an index of storm activity, $\mathbf{S}_{S, J}$ was replaced by its average.

[59] 11. It is well established that the pattern of the daily variation changes with season and undergoes other long-term changes. We therefore see the primary application of the proposed method in computing the index over a window of 2 months. Assuming daily periodicity of $P_{s, t}$ over the period of 1 year is more questionable, but the WISA computed over 1 year is still remarkably close to the standard Dst. A detailed comparison is presented in section 6 .

[60] 12. The complexity of MODWT is $O\left(n \log _{2}(n)\right)$. On Sun V2-40, with two $1.28 \mathrm{GHz}$ processors and $8192 \mathrm{MB}$ of 
DIFFERENCES BETWEEN DSt AND WISA FOR 2001
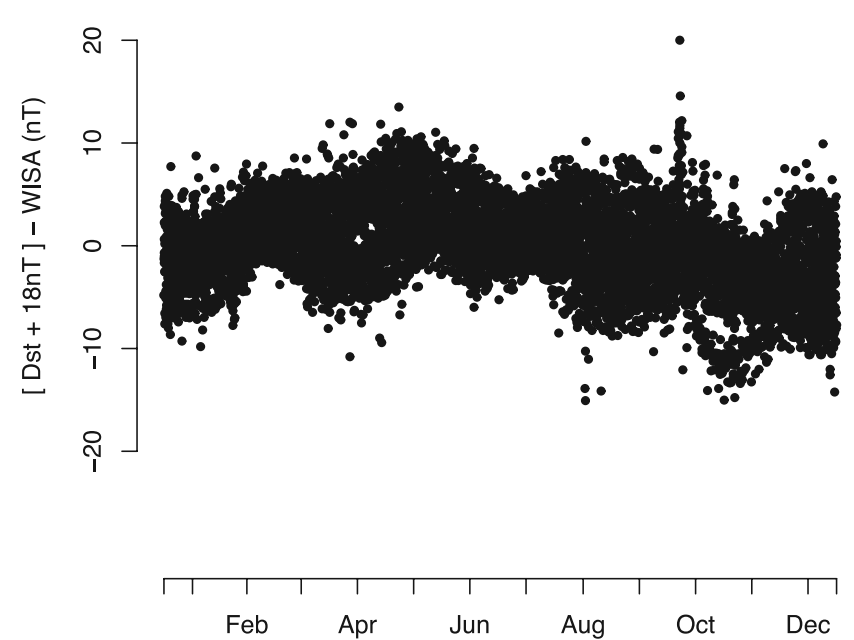

Figure 4. Differences between Dst $(+18 \mathrm{nT})$ and WISA, January-December 2001.

RAM, it takes slightly under an hour to obtain WISA for $N=$ 1 year, and slightly under $3 \min$ for $N=2$ months.

\section{Comparison to Dst}

[61] The objective of this section is to compare the new WISA index to the Dst index. There are several sources of differences:

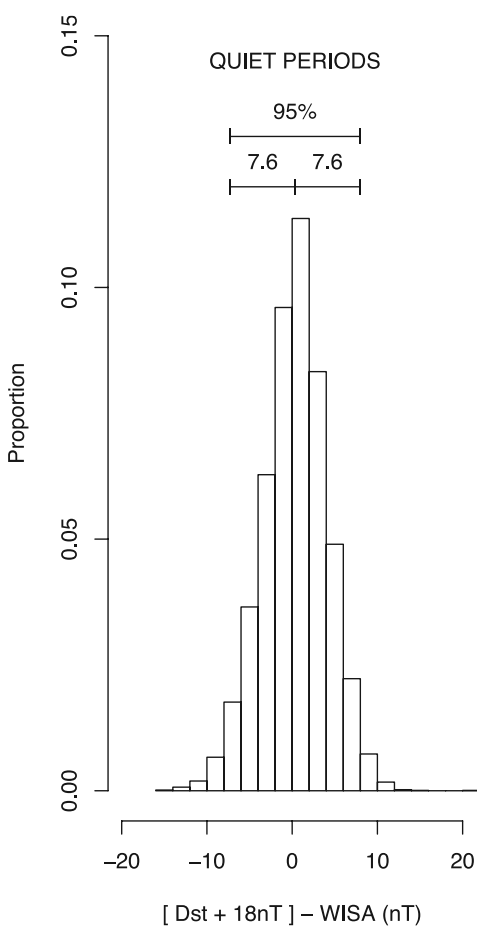

[62] 1. WISA and Dst are calculated in fundamentally different way.

[63] 2. Dst is an hourly index and WISA is a 1-min index. To obtain hourly values of WISA we used 1-min averages from INTERMAGNET's CDs (which are themselves averages of raw data recorded with $5 \mathrm{~s}$ frequency) and then averaged the 60 values of 1-min WISA following the time for which Dst is reported.

[64] 3. Even the best quality records contain missing values. We interpolated the missing via linear interpolation. A different interpolation technique would produce slightly different WISA values. We do not know how missing values are handled to produce Dst.

[65] Ideally, we would like to compare WISA to Dst with respect to differences arising from the different methodology, i.e., from source 1. This source cannot however be separated from sources 2 and 3 and keeping this in mind we will argue that the contribution of source 1 is negligible; that is, over a period of 1 year our technique can automatically reproduce $D s t$ up to the accuracy determined by the preprocessing of the raw data.

[66] In the comparison below we use the final 1-hour Dst index and WISA computed from 1-min values of the $\mathrm{H}$ component for Hermanus, Honolulu, Kakioka and San Juan (Dst stations). We consider the time period JanuaryDecember 2001, which contains a few very strong storms with the dynamic range approaching $500 \mathrm{nT}$. By construction, the average of the WISA is zero, compare comment 3 ) in section 5. The Dst is computed over longer periods of time and its average is not zero for any subperiod (but is close to zero). To make the comparison possible, we subtracted the average value of the Dst for the periods over which we compare it to WISA. Specifically, when using the Dst over

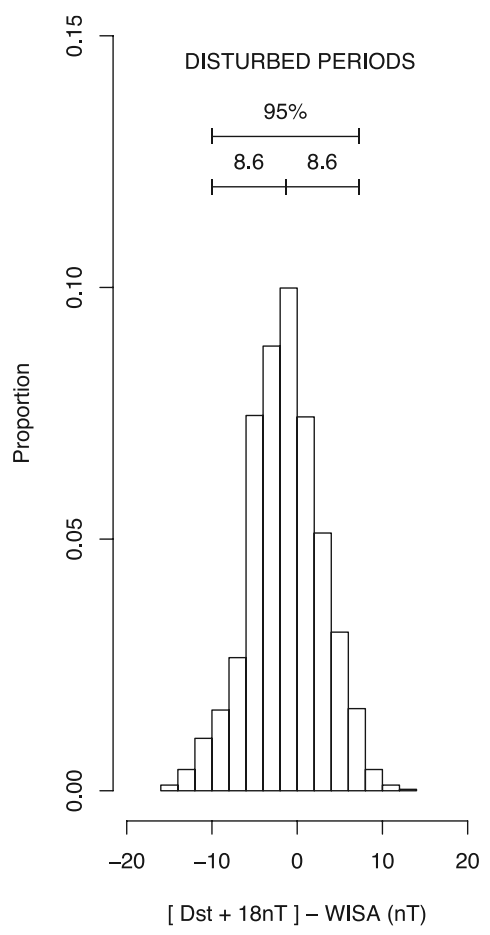

Figure 5. Histograms of differences between Dst (+18 nT) and WISA for "quiet" and "disturbed" periods, January-December 2001. 


\section{STORM ACTIVITY INDICES}

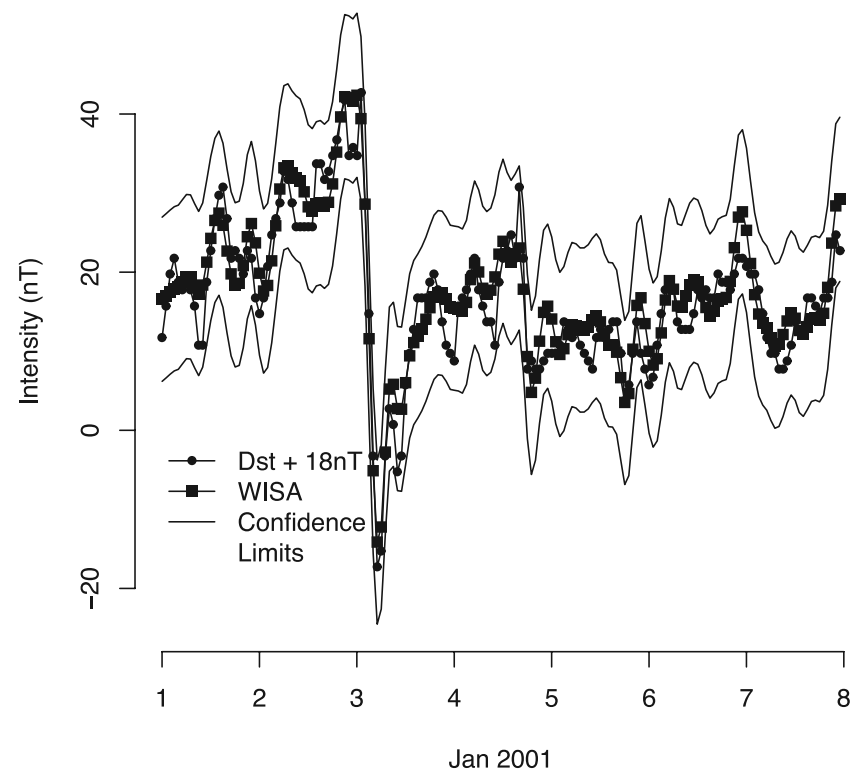

Figure 6. Dst, WISA, and $95 \%$ confidence intervals for hourly WISA for the first week of 2001.

the whole year 2001, we added $18 \mathrm{nT}$ to it to make its average for 2001 zero. For the subperiods of 2 months, different constants had to be added. For example, for the period of March-April, which contains a very strong storm, we added $31 \mathrm{nT}$ to the Dst to make its average over this 2-month period zero.
[67] Figure 3 shows the Dst index (Figure 3, bottom) and the hourly averages of WISA (Figure 3, top). The two indices look very similar. In Figure 4, the differences between Dst and WISA are plotted and are seen to be generally smaller than $10 \mathrm{nT}$. These differences tend to be slightly larger during disturbed period $(D s t<-30 \mathrm{nT})$ than during quiet periods (Dst $\geq-30 \mathrm{nT})$. This point is illustrated in Figure 5. The physical interpretation of the larger spread of differences for disturbed conditions requires additional study with larger data sets, but it can be conjectured that our approximation of the diurnal component is more accurate during quiet periods; during disturbed periods this component appears less stable. The differences for both the disturbed and calm periods have an error-type distribution, indicating that they may be attributable to chance errors rather than a systematic bias.

[68] As explained above, the averaging of WISA to obtain an hourly index introduces some arbitrary variability. To assess this variability, we computed the differences

$$
\Delta I_{t}=I_{t}-I_{t-1}, \quad t=1,2, \ldots,\lfloor N / 60\rfloor-1 .
$$

The sample standard deviation of these differences, denoted $s_{\Delta I}$ is a measure of variability due to averaging over one hour intervals. Since the differences are approximately normally distributed, the $100(1-\alpha) \%$ confidence interval for the hourly WISA can be defined as

$$
\left(I_{t}-z_{1-\alpha / 2} s_{\Delta I}, I_{t}+z_{1-\alpha / 2} s_{\Delta I}\right),
$$

where $z_{\beta}$ denotes $\beta$ th quantile of the standard normal distribution. For the whole of 2001, $s_{\Delta I}=5.20 \mathrm{nT}$, and

STORM ACTIVITY INDICES FOR MAR-APR 2001
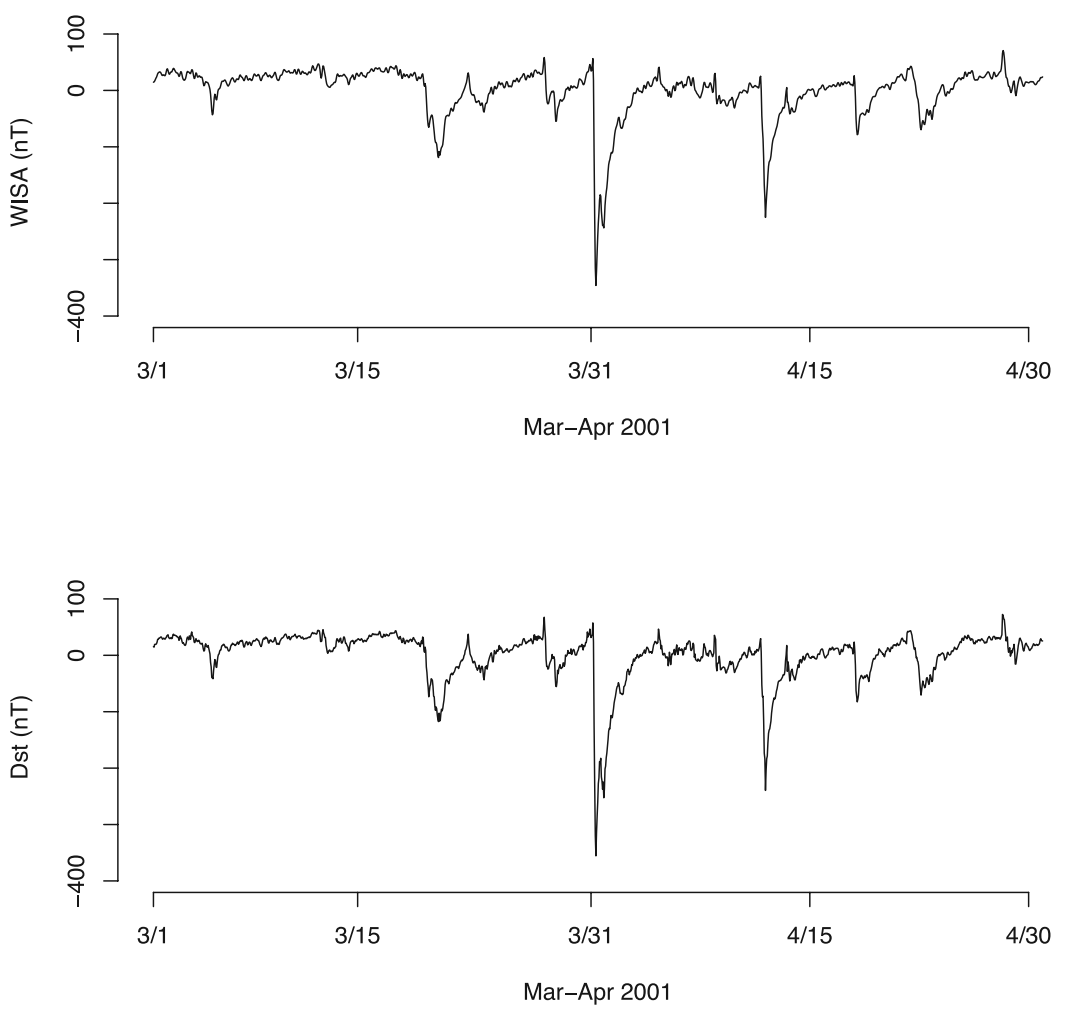

Figure 7. Hourly values of WISA and Dst, March-April 2001. 


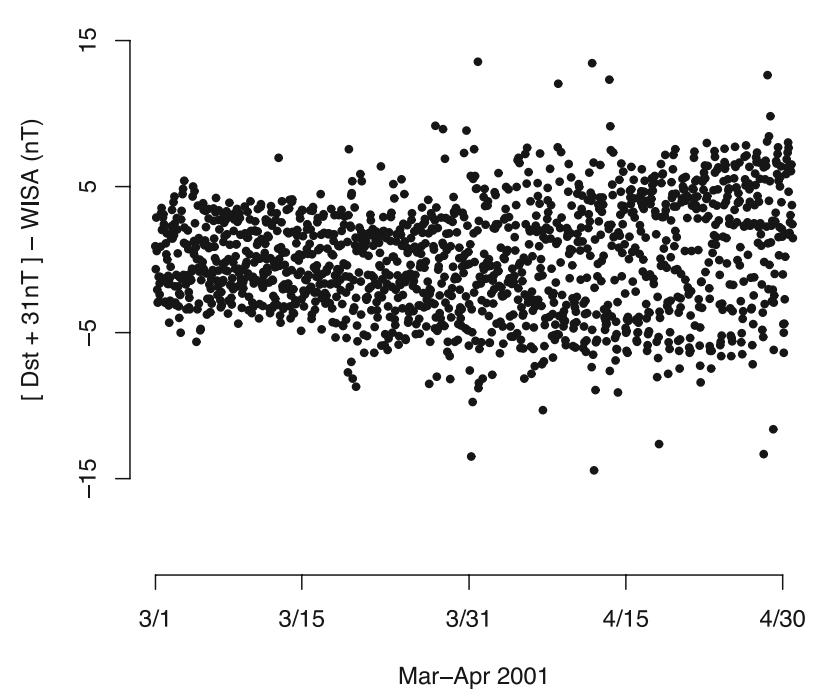

Figure 8. Differences between Dst $(+31 \mathrm{nT})$ and WISA, March-April 2001.

$97.72 \%$ of the Dst values are within the $95 \%$ confidence limits. Figure 6 shows the 95\% confidence intervals together with the two indices for the first week of January. The graphs have a similar appearance for the remaining weeks of 2001.

[69] We also compared hourly WISA computed over 2-month periods in 2001 to the Dst. Graphs for the most

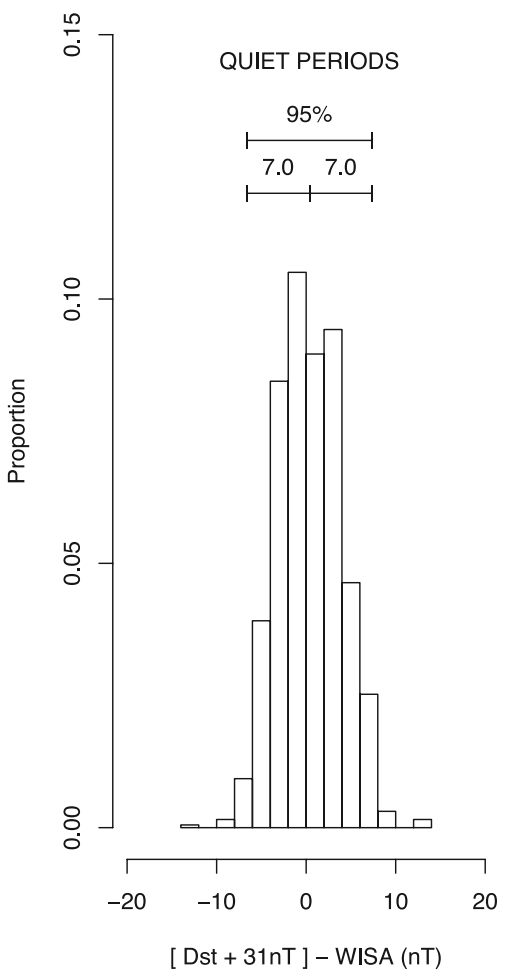

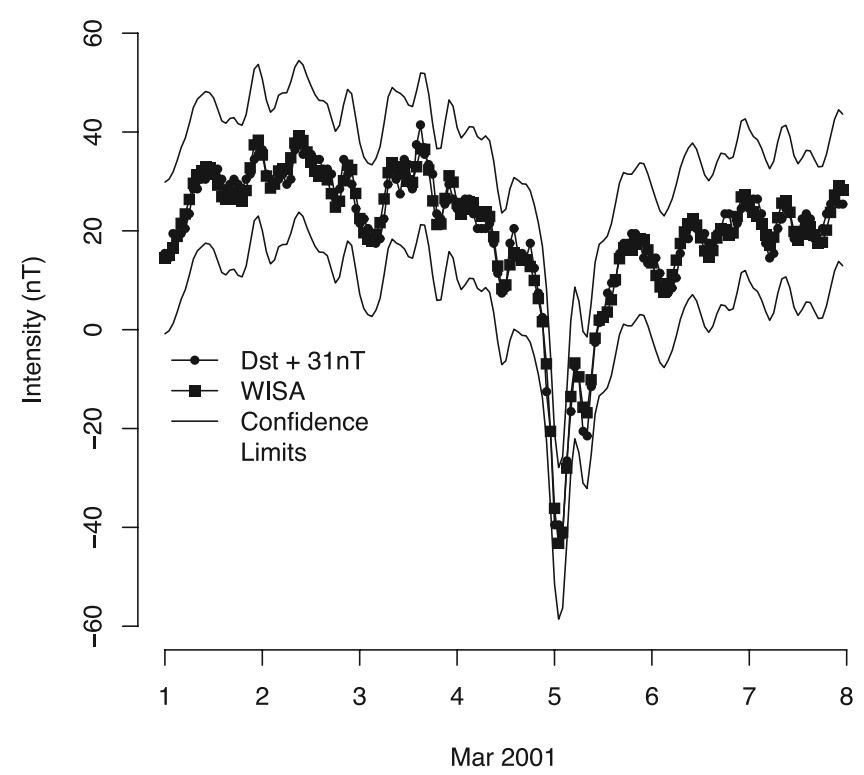

Figure 10. Dst, WISA, and $95 \%$ confidence intervals for hourly WISA for the first week of March.

stormy period, March-April, are presented in Figures 7-10. For this period, all Dst values are within the 95\% confidence limits. The increasing spread of differences visible in Figure 8 is due to a very strong storm in the middle of that period. As for the WISA computed from 1 year worth of data, the variability due to the averaging of

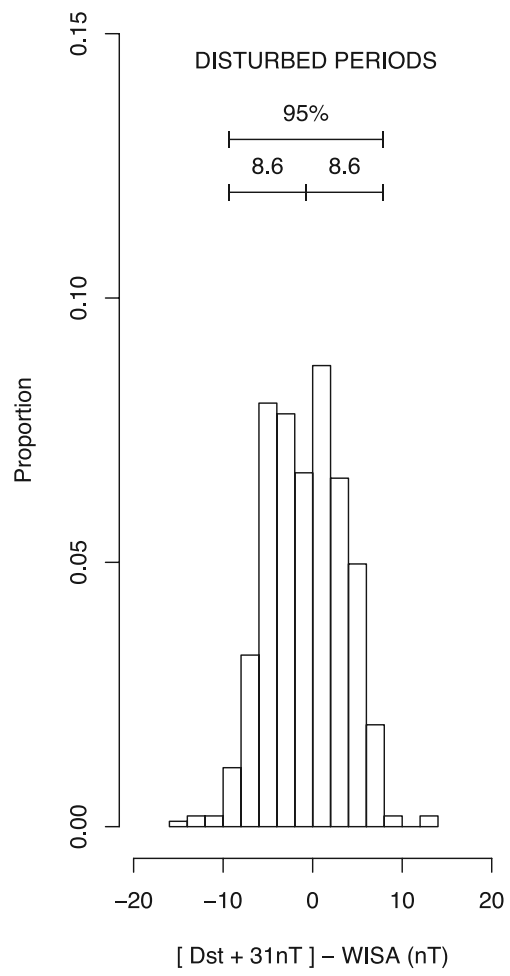

Figure 9. Histograms of differences between Dst (+31 nT) and WISA for "quiet" and "disturbed" periods, March-April 2001. 
the 1-min WISA is statistically greater than the differences between the hourly WISA and the Dst. Perhaps more importantly, the difference between the WISA and the Dst, which is typically about $5 \mathrm{nT}$, is negligible compared to the dynamic range of a storm which is several hundred nanoteslas.

\section{Conclusions}

[70] We propose an automatic procedure for calculating an index of geomagnetic storm activity which uses scale time-dependent decomposition provided by the Maximum Overlap Discrete Wavelet Transform and statistical filtering techniques. We use the wavelet thresholding to remove the noise and a median-based filter to remove the periodic component. The procedure produces a 1-min index and requires only a recent short stretch of data. It replaces the Dst determination of quiet days involving subjective human selection by robust statistical estimation of the diurnal component. When the $\mathrm{H}$ components of the four Dst stations are provided as input, the hourly averages of the new index are statistically indistinguishable from the Dst values.

[71] The procedure can take magnetometer data from different stations as input thus allowing the study of the effect of the choice of the stations on the index of storm activity. Such a study is however beyond the scope of this work. The proposed procedure is flexible and amenable to various modifications and can potentially serve as a useful tool in the study of the magnetic storms. Its full automation has a substantial potential for operational purposes.

[72] Research is ongoing to investigate both the shortterm storm related wavelet decompositions and the longterm seasonal and solar cycle dependencies of the other components in equation (2).

[73] Acknowledgments. This research was supported by NSF grant DMS0413653 and NASA grant NNG05GJ48G. The magnetometer data were provided by INTERMAGNET.
[74] Wolfgang Baumjohann thanks Zoltan Voros and another referee for their assistance in evaluating this paper.

\section{References}

Brockwell, P. J., and R. A. Davis (2002), Introduction to Time Series and Forecasting, 2nd ed., Springer, New York.

Campbell, W. H. (1999), Comment on "Current understanding of magnetic storms: Storm -substorm relationship" by Y. Kamide et al., J. Geophys. Res., 104, 7047.

Cornish, C. R., C. S. Bretherton, and D. B. Percival (2004), Wavelet analysis of marine atmospheric boundary layer turbulence during epic, technical report, Univ. of Wash., Seattle. (Available at http:// faculty.washington.edu/dbp/)

Feldstein, Y. I., et al. (2005), Self-consistent modeling of the large-scale distortions in the geomagnetic field during the 24-27 September 1998 major magnetic storm, J. Geophys. Res., 110, A11214, doi:10.1029/ 2004JA010584.

Kamide, Y., W. Baumjohann, I. A. Daglis, W. D. Gonzalez, M. Grande, and J. A. Joselyn (1998), Current understanding of magnetic storms: Stormsubstorm relationship, J. Geophys. Res., 103, 17,705.

Kivelson, M. G., and C. T. Russell (Eds.) (1997), Introduction to Space Physics, Cambridge Univ. Press, New York.

Kokoszka, P., I. Maslova, J. Sojka, and L. Zhu (2006), Probability tails of wavelet coefficients of magnetometer records, J. Geophys. Res., 111, A06202, doi:10.1029/2005JA011486.

Overland, J. E., M. C. Spillane, D. B. Percival, M. Wang, and H. O. Mofjeld (2004), Seasonal and regional variation of pan-arctic surface air temperature over the instrumental record, J. Clim., 17, 3263-3282.

Parkinson, W. D. (1983), Introduction to Geomagnetism, Scott. Acad. Press, Edinburgh.

Percival, D. B., and A. T. Walden (2000), Wavelet Methods for Time Series Analysis, Cambridge Univ. Press, New York.

Rostoker, G. (2000), Effects of substorms on the storm-time ring current index Dst, Ann. Geophys., 18, 1390.

Sugiura, M. (1964), Hourly values of equatorial Dst for the IGY, Ann. Int. Geophys. Year, 35(9).

Whitcher, B., S. D. Byers, P. Guttorp, and D. B. Percival (2002), Testing for homogeneity of variance in time series: Long memory, wavelets, and the Nile River, Water Resour. Res., 38(5), 1054, doi:10.1029/ 2001 WR000509.

A. Jach and P. Kokoszka, Department of Mathematics and Statistics, Utah State University, 3900 Old Main Hill, Logan, UT 84322, USA. (ajach@cc.usu.edu; piotr.kokoszka@usu.edu)

J. Sojka and L. Zhu, Department of Physics and Center for Atmospheric and Space Science, Utah State University, 4405 Old Main Hill, Logan, UT 84322, USA. (fasojka@sojka.cass.usu.edu; zhu@cc.usu.edu) 